\title{
Impact of Maternal Human Immunodeficiency Virus Infection on Birth Outcomes and Infant Survival in Rural Mozambique
}

\author{
Denise Naniche,* Azucena Bardají, María Lahuerta, Anna Berenguera, Inacio Mandomando, Sergi Sanz, \\ John J. Aponte, Betuel Sigauque, Pedro L. Alonso, and Clara Menéndez \\ Barcelona Center for International Health Research, Hospital Clinic, Institut d'Investigacions Biomèdiques August Pi i Sunyer, Universitat \\ de Barcelona, Barcelona, Spain; Centro de Investigaçâo em Saúde de Manhiça, Manhiça, Mozambique; Instituto Nacional de Saúde \\ Maputo, Mozambique; Faculdade de Medicina da Universidade Eduardo Mondlane, Maputo, Mozambique
}

\begin{abstract}
We assessed the effect of maternal human immunodeficiency virus (HIV) infection on birth outcomes and infant survival in rural Mozambique. Pregnant women attending the antenatal clinic were recruited. These women and their infants were followed-up for one year. Birth outcomes were assessed at delivery and infant HIV status was determined at 1 and 12 months of age. Women positive for HIV were more likely to have anemia at delivery than women negative for HIV $(51.3 \%$ versus $35.4 \% ; P<0.001)$. Infants born to HIV-positive mothers had a significantly higher postneonatal mortality rate than infants born to HIV-negative mothers $(7.8 \%$ versus $1.9 \% ; P<0.001)$. The rate of transmission of HIV by breastfeeding during the first year of life was $15.1 \%(95 \%$ confidence interval $=$ CI $7.6-22.4)$. Assessment of the impact of HIV infection on birth outcomes in rural Africa is essential for tailoring public health measures to reduce mother-to-child transmission of HIV and excess infant mortality.
\end{abstract}

\section{INTRODUCTION}

Sub-Saharan Africa harbors more than two-thirds of the world's 33.2 million persons infected with human immunodeficiency virus (HIV) and $80 \%$ of the world's HIV-infected women. ${ }^{1}$ In parts of southern Africa, more than $30 \%$ of pregnant women attending antenatal clinics are infected with HIV, thus making HIV infection one of the most common complications of pregnancy in sub-Saharan Africa. ${ }^{1}$

With successful interventions, mother-to-child transmission (MTCT) of HIV has been reduced to less than $2 \%$ in developed countries. ${ }^{2}$ However, in untreated populations, MTCT of HIV during pregnancy, delivery, and breastfeeding still occurs at an approximate overall rate of $25-40 \%, 3,4$ and accounts for almost 420,000 new HIV infections in children and 270,000320,000 pediatric deaths annually. ${ }^{1,5}$ Until 2004, single-dose intrapartum and neonatal nevirapine (sd-NVP) was the recommended regimen by the World Health Organization to prevent MTCT of HIV among women without access to antiretroviral therapy. Preventive MTCT programs with an sd-NVP have been shown to decrease perinatal HIV transmission to $8 \%$ in controlled clinical trial settings. ${ }^{6}$ However, there is great concern about the rapid development of resistance. In addition, in predominantly breastfeeding populations of subSaharan Africa, most MTCT of HIV still occurs during the postnatal period. ${ }^{4}$ Currently, MTCT prevention programs in sub-Saharan African countries include zidovudine and lamivudine during the final weeks of pregnancy and sd-NVP at delivery. In addition, the newborn receives sd-NVP at birth and zidovudine for seven days. ${ }^{7}$ Nevertheless, effectiveness of these strategies relies on the great challenge of availability of the drugs and compliance with them, given that these preventive regimens are prolonged and unsupervised.

Several studies from the Africa have reported that HIVinfected pregnant women are at increased risk of adverse

\footnotetext{
* Address correspondence to Denise Naniche, Barcelona Center for International Health Research, Hospital Clinic, Institut d'Investigacions Biomèdiques August $\mathrm{Pi}$ i Sunyer, Universitat de Barcelona, C/Villarroel, 170, Barcelona 08036, Spain. E-mail: dsuzanne@clinic.ub.es
}

pregnancy outcomes such as spontaneous abortion, stillbirths, and preterm labor. ${ }^{8-10}$ However, this analysis is complicated by many factors associated with HIV infection and poor pregnancy outcomes such as malnutrition, anemia, and other frequent infections such as syphilis or malaria. ${ }^{11}$ These factors may contribute to the observation that the association between HIV infection and adverse pregnancy outcomes is stronger in women from developing countries. ${ }^{12,13}$

Maternal HIV infection has also been associated with an increased risk of infant death., ${ }^{5,15}$ It is well documented that up to $35 \%$ of HIV-infected infants may die before the first year of age, ${ }^{1}$ but HIV-negative children born to HIV-infected mothers are also at high risk of mortality. ${ }^{15}$

There have been few studies characterizing the impact of HIV infection during pregnancy on the mother and her infant ${ }^{5,16,17}$ and even fewer from rural African settings. ${ }^{14,18}$ The main aim of this study was to assess the impact of HIV infection on birth outcomes and infant survival in a rural area of southern Mozambique. Furthermore, the study also evaluated the effect of unsupervised sd-NVP administration for prevention of MTCT of HIV on HIV RNA viral load at delivery and the prevalence of NVP resistance mutations.

\section{METHODS}

Study population. The study was conducted from August 2003 through October 2006 at the Manhiça District Hospital in Manhiça District, Maputo Province, in southern Mozambique. The Centro de Investigaçao em Saúde de Manhiça has been conducting continuous demographic surveillance in the Manhiça District since 1996 that covers a population of 82,000 persons. ${ }^{19}$

The prevalence of HIV infection in pregnant women attending the antenatal clinic in 2004 was approximately $21 \%{ }^{20}$ During the study, highly active anti-retroviral treatment was not available, and the national policy on prevention of MTCT of HIV relied upon the self-administration of sd-NVP to the mother at the onset of labor and to the newborn within the first 72 hours of life. Because NVP uptake by the mother was unsupervised, maternal adherence to the sd-NVP regimen was ascertained by interview prior to delivery. Breastfeeding was common with more than $90 \%$ of children in the area 
breastfeeding during the first six months of age (Menendez C, unpublished data).

Study design. This study was integrated into a randomized, double-blind, placebo-controlled trial (RCT) of two-dose intermittent preventive treatment (IPTp) with sulfadoxinepyrimethamine (SP) for malaria prevention in pregnancy (trial registration no. NCT00209781). All study participants had been given a long-lasting insecticide treated bed net through the antenatal clinic. Details of the RCT design are given elsewhere. ${ }^{20}$ Pregnant women participating in the RCT were invited to participate in the study of HIV, birth outcomes, and MTCT $^{21}$ if they accepted HIV testing at the voluntary counseling and testing (VCT) center. The current study started after the RCT had been initiated. Thus, baseline CD4 cell counts and HIV RNA viral load data are only available for the HIV-positive women enrolled during the second half of the trial, although HIV RNA viral load data is available for all HIV-positive women at delivery and one-month postpartum. The study was reviewed and approved by the National Mozambican Ethics Committee and the Hospital Clinic of Barcelona Ethics Review Committee. ${ }^{20,21}$

Follow up and sample collection. After written informed consent was obtained, a capillary blood sample was collected as part of the routine antenatal care to assess anemia and for syphilis screening. The HIV-1 viral status and the CD4 cell counts of the mother were determined at time of enrollment after voluntary counseling. Women with indeterminate results were invited to return one month later for testing. The HIV RNA viral load and CD4 cell counts were determined at enrollment, at delivery, and at one-month postpartum. Recent HIV infections in the mother (less than six months prior to enrollment) were determined on a subset of women (80 of 93) from whom a sufficient blood sample was available at the time of the enrollment visit. The mid-upper arm circumference (MUAC) was also measured to assess the nutritional status. All women were encouraged to deliver at Manhiça District Hospital.

At the time of delivery, venous blood was collected from the mother and the umbilical cord for hematologic and parasitologic determinations, and a placental biopsy sample was collected for malaria examination. A placental blood sample was collected for placental HIV-1 RNA assays from the women enrolled during the second half of the trial. Comparison of peripheral malaria at delivery and placental malaria between HIV-positive and HIV-negative women is only shown on the group of women who received placebo because the IPTp with SP was found to be effective in reducing the risk of malaria infection in the mother in the main RCT. ${ }^{20}$ Newborns were weighed on a digital scale, accurate to the nearest gram.

The mother was examined 4-6 weeks postpartum for hematologic, parasitologic, and viral determinations. Infants were visited at 4-6 weeks and 12 months of age. At each visit, infants were examined and weighed, and a capillary blood sample was collected for hematologic, parasitologic, and viral determinations. An HIV-DNA polymerase chain reaction (PCR) was carried out at these two visits. To confirm HIV status, children with positive HIV-DNA PCR results at any of these visits were invited to return for a second test one month later. The HIV-DNA PCR was performed at 12 months of age to confirm HIV infection at one month, if not confirmed earlier, and to identify transmission caused by breastfeeding. According to national guidelines, at 18 months of age, children were invited to return for serologic HIV testing at the VCT program.

Laboratory methods. Maternal HIV status was assessed using the Determine HIV-1/2 Rapid Test (Abbott Laboratories, Abbott Park, IL) and positive results were confirmed by the Uni-Gold Rapid Test (Trinity Biotech Co., Wicklow, Ireland). The HIV status of the infants was assessed using the Amplicor HIV-1 DNA-PCR kit (Roche Diagnostics, Branchburg, NJ). Recent maternal HIV infections were determined using the Calypte HIV-1 BED Incidence Enzyme Immunoassay (Calypte Biomedical Corporation, Lake Oswego, OR).

Maternal HIV RNA viral load was determined from cryopreserved peripheral and placental plasma using the Amplicor HIV-1 Monitor assay version 1.5 (Roche Diagnostics). The assay has a sensitivity of 400 copies/mL. For the purpose of analyses, plasma HIV-1 RNA concentrations below the limit of detection were assigned the value of 200 copies $/ \mathrm{mL}$. The CD4 cell counts were determined by flow cytometry after staining of whole blood with fluorochrome-labeled antibodies to CD3, CD8, and CD4 cells and acquisition using Truecount tubes (Becton Dickinson, Franklin Lakes, NJ). Mutations known to confer resistance to nevirapine were assessed after reverse transcription-PCR and sequencing of codons 26-245 of the HIV-1 reverse transcriptase gene from a subsample of 41 women one month after delivery with primers and conditions as described previously. ${ }^{22}$ The Stanford University HIV Drug Resistance database (aIgProgram) (http://hivdb.stanford.edu) was used to determine resistance-associated mutations.

Determination of maternal and infant anemia was performed by assessment of packed cell volume (PCV) in a microhematocrit centrifuge and reading in a Hawksley hematocrite reader (Hawksley-Gelman, Lancing, United Kingdom). Syphilis screening was performed with the rapid plasma reagin test, (Syphacard; Burroughs-Wellcome, Research Triangle Park, NC).

Statistical methods and definitions. Malaria infection was defined as any density of asexual Plasmodium falciparum parasites in a blood smear. Malnutrition was defined as MUAC $\leq$ $22 \mathrm{~cm}$. Anemia in pregnant women was defined as a PCV less than $33 \%$ and severe anemia as a PCV less than $21 \%$. Placental malaria was defined as the presence of parasites and/or pigment in a histologic examination. Fetal anemia was defined as a PCV less than $37 \%$ in cord blood and/or less than $42 \%$ in the newborn peripheral blood. Perinatal HIV infection was defined as a positive HIV DNA-PCR result at 4-6 weeks of age. Infection with HIV during breastfeeding was defined as a negative HIV DNA-PCR result at 4-6 weeks of age and a positive HIV DNA-PCR result at 12 months of age. A recent HIV infection in the mother was considered as an infection occurring within the previous six months before the enrollment visit. Anemia in infants was defined as a PCV less than $33 \%$, and low birth weight as a birth weight lower than 2,500 grams. Early neonatal mortality refers to a death of a live-born baby within the first seven days of life, and late neonatal mortality covers the time between 7 and 29 days of life. Post neonatal death includes the remaining 11 months of the first year of life. ${ }^{23}$

Microsoft FoxPro version 5.0 (Microsoft Corp., Redmond, WA) was used for data entry, validation, and cleaning of data, and statistical analysis was performed with STATA release 9 (StataCorp, College Station, TX). Proportions for categorical variables were assessed using the chi-square test or Fisher's 
exact test where appropriate. The Wilcoxon rank sum test was used to compare independent continuous variables with non-normal distribution. McNemar's chi-square test and the Wilcoxon signed rank were used for analysis of paired data for categorical and continuous variables, respectively. The Spearman correlation was used to assess correlations between non-normally distributed variables. Probability of MTCT during the first year of life was calculated as 1 - (negative proportion at 1 month $\times$ negative proportion at 12 month).

\section{RESULTS}

A total of 1,030 women were enrolled in the RCT, ${ }^{20}$ of which 870 women accepted HIV testing. Of these women, 207 were HIV positive and 660 were HIV negative.

Maternal outcomes in HIV-positive and HIV-negative pregnant women. Comparison of demographic and analytical parameters between HIV-positive and HIV-negative women are shown in Table 1 . The average gestational age at enrollment was 24 weeks. The mean \pm SD time between HIV testing and delivery was $3.7 \pm 1$ month. The HIV-positive women were significantly more likely to have anemia at delivery $(51.3 \%$ [102 of 199] in HIV-positive women versus 35.4\% [224 of 633] in HIV-negative women) $(P<0.001)$ (Table 1$)$. There was no evidence of a higher proportion of HIV-positive women with peripheral malaria parasitemia at delivery $(19.8 \%, 17$ of 86$)$ than HIV-negative women $(13.3 \%, 43$ of 324$)(P=0.130)$. There was also a trend for a higher proportion of HIV-positive women with a positive syphilis test result (15\% [31 of 207] versus $10.9 \%$ [72 of 660 ] in HIV-negative women) $(P=0.115)$. The HIV-positive women had a higher prevalence of malnutrition compared with HIV-negative women (3.7\% [6 of 164] versus $1.3 \%$ [7 of 524]) $(P=0.057)$.

Birth outcomes in HIV-positive and HIV-negative pregnant women. Excluding 7 second live-born twins and 2 live-born triplets, there were 839 live-born singleton babies, 199 of whom were born to HIV-positive women and 640 of whom were born to HIV-negative women. Median birth weight tended to be lower in infants born to HIV-positive mothers versus HIV-negative mothers (3,000 g versus 3,040 g; $P=0.064)$. Although not statistically significantly different $(P=0.156)$, the proportion of low birth weight babies born to
HIV-positive women was also higher as compared with HIVnegative women $(13.8 \%$ versus $10.1 \%$ ) (Table 2$)$.

There was a 1.5-fold higher prevalence of anemia at 1 month of age among infants born to HIV-positive women (20\%, 32 of 160) compared with infants born to HIV-negative women $(13.8 \%, 71$ of 514$)(P=0.058)$ (Table 2). Malaria parasitemia at one month of age was similar in both groups of infants $(P=0.640)$. There was no evidence of an association between spontaneous abortion, premature delivery, stillbirth or neonatal death and HIV status of the mother (Table 2).

Overall infant mortality was 2.3 -fold higher in babies born to HIV-positive women than those born to HIV-negative women $(P=0.002)$ (Table 2$)$. When separated into neonatal death and post-neonatal death, infants born of HIV-positive mothers had a higher post neonatal mortality rate $(7.8 \%, 15$ of 193) than those born to HIV-negative mothers $(1.9 \%, 12$ of 623) $(P<0.001)$. Early and late neonatal death were similar in infants born to HIV-positive women and to HIV-negative women. The lost to follow-up was low and equally distributed between infants born to HIV-positive women and those born to HIV-negative women $(P=0.86$, by log rank test for equality of lost to follow-up). Potential bias could be a slight underestimation of the post neonatal mortality.

Viral and immunologic parameters of HIV infection in pregnant women. The proportion of women refusing the test and the proportion of women positive for HIV changed over the study duration. During the first half of recruitment (from August 2003 through May 2004), the prevalence of HIV was $18.4 \%$ (78 of 423 ) (95\% confidence interval [CI] $=14.7-22.0)$, with $17.9 \%$ (92 of 515) of women refusing VCT. However, during the second half of recruitment (from May 2004 through April 2005), the prevalence of HIV increased to $29 \%$ (130 of 447$)(95 \% \mathrm{CI}=24.9-33.4)$, and the percentage of women refusing VCT decreased to $13.2 \%$ (68 of 515). The prevalence of HIV was significantly different by age group (18.2\% [50 of 274 ] in women less than 20 years of age, $27.4 \%$ [113 of 413 ] in women 20-30 years of age, and $24.4 \%$ [ 44 of 280] in women more than 30 years of age; $P=0.026$, by Fisher's exact test).

Characterization of the proportion of women with recent HIV infection ( $<6$ months) was performed on a subset of women at the time of the enrollment visit (as described in the Materials and Methods). Fifteen (18.8\%) of 80 women

TABLE 1

Characteristics of 207 HIV-positive and 660 HIV-negative pregnant women enrolled in the study*

\begin{tabular}{|c|c|c|c|c|c|}
\hline Characteristic & \multicolumn{2}{|c|}{$\mathrm{HIV}+$} & \multicolumn{2}{|c|}{ HIV- } & $P^{\dagger}$ \\
\hline Age, years & \multicolumn{2}{|c|}{$23(20-29)$} & \multicolumn{2}{|c|}{$22(18-28)$} & $0.005 末$ \\
\hline \multicolumn{6}{|l|}{ Gravidity } \\
\hline Primigravid & $53 / 207$ & $(25.6)$ & $184 / 660$ & $(27.9)$ & 0.814 \\
\hline Secundigravid & $46 / 207$ & $(22.2)$ & $142 / 660$ & $(21.5)$ & \\
\hline Multigravid & $108 / 207$ & $(52.2)$ & $334 / 660$ & $(50.6)$ & \\
\hline Literate & $73 / 206$ & $(35.4)$ & $273 / 659$ & $(41.4)$ & 0.126 \\
\hline Malnutrition at enrollment $\S$ & $6 / 164$ & $(3.7)$ & $7 / 524$ & $(1.3)$ & 0.057 \\
\hline RPR & $31 / 207$ & $(15)$ & $72 / 660$ & $(10.9)$ & 0.115 \\
\hline $\mathrm{PCV}<33 \%$ at delivery & $102 / 199$ & $(51.3)$ & $224 / 633$ & $(35.4)$ & $<0.001$ \\
\hline Peripheral malaria at delivery $\mathbb{I}$ & $17 / 86$ & $(19.8)$ & $43 / 324$ & $(13.3)$ & 0.130 \\
\hline Placental malaria\# & $40 / 75$ & $(53.3)$ & $143 / 274$ & $(52.2)$ & 0.861 \\
\hline
\end{tabular}


TABLE 2

Adverse pregnancy and neonatal outcomes by HIV status of the mother*

\begin{tabular}{|c|c|c|c|c|c|}
\hline Outcome & \multicolumn{2}{|c|}{ HIV+ mothers $(n=199)$} & \multicolumn{2}{|c|}{ HIV-mothers $(n=640)$} & $P \dagger$ \\
\hline Birth weight, grams & \multicolumn{2}{|c|}{$3,000(2,600-3,300)$} & \multicolumn{2}{|c|}{$3,040(2,780-3,340)$} & $0.064 \S$ \\
\hline Low birth weight infants & 27/196 & $(13.8)$ & $64 / 631$ & (10.1) & 0.156 \\
\hline Fetal anemiaf & $14 / 175$ & (8) & $45 / 554$ & $(8.1)$ & 0.960 \\
\hline Infant anemia at one month & $32 / 160$ & (20) & $71 / 514$ & $(13.8)$ & 0.058 \\
\hline Infant parasitemia at one month & $2 / 168$ & (1.2) & $4 / 524$ & $(0.8)$ & $0.640 \#$ \\
\hline Spontaneous abortion & $4 / 207$ & (1.9) & $5 / 660$ & $(0.8)$ & $0.229 \#$ \\
\hline Pre-term & $10 / 167$ & (6) & $19 / 522$ & (3.6) & 0.190 \\
\hline Stillbirth & $4 / 203$ & (2) & $15 / 655$ & (2.3) & $1 \#$ \\
\hline Infant mortality & 21/199 & $(10.6)$ & $29 / 640$ & (4.5) & 0.002 \\
\hline Early neonatal death & $4 / 199$ & (2) & $13 / 640$ & (2) & $1 \#$ \\
\hline Late neonatal death & $2 / 195$ & (1) & $4 / 627$ & $(0.6)$ & $0.630 \#$ \\
\hline Post-neonatal death & $15 / 193$ & (7.8) & $12 / 623$ & (1.9) & $<0.001$ \\
\hline
\end{tabular}

*Values are median (interquartile range) or no. positive/no. tested (\%). HIV = human immunodeficiency virus; IQR = interquartile range.

$\dagger$ Proportions were compared using the chi-square test unless otherwise stated.

$\doteqdot$ Calculated from live births (excludes spontaneous abortions and stillbirths).

$\S$ By Wilcoxon rank sum test.

If Fetal anemia was defined as a packed cell volume $<37 \%$ in cord blood $\leq 42 \%$ in newborn peripheral blood

\#By Fisher's exact test.

analyzed (95\% CI = 10-27.5) had an HIV infection contracted within the previous six months.

The median peripheral HIV RNA viral load increased between enrollment to one-month post-delivery, although the difference did not reach statistical significance $(P=0.215$, by signed rank test for paired observations) (Table 3 ). The proportion of HIV-positive women with HIV RNA viral load greater than 10,000 copies $/ \mathrm{mL}$ was similar at the time of enrollment and at delivery $(59.1 \%$ and $60.3 \%$, respectively). However this value increased to $69.5 \%$ one month after delivery ( $P=0.014$, by McNemar's chi square test for paired observations). Placental HIV RNA viral load was measured in a subset of 57 women and showed a median of 8,728 copies/ $\mathrm{mL}$ (interquartile range $[\mathrm{IQR}]=2,098-46,467$ ), which was lower than that in peripheral blood $(17,515$ copies $/ \mathrm{mL}, \mathrm{IQR}=$ $4,800-69,492)$, although not significantly different $(P=0.240)$ (Table 2).

Median CD4 cell counts increased significantly in the interval between enrollment and one-month post-delivery (Table 3 ). Furthermore, at time of enrollment, $28 \%$ of the women had CD4 cell counts less than $350 / \mu \mathrm{L}$, whereas one-month after

TABLE 3

Viral and immunologic parameters of HIV-positive women*

\begin{tabular}{|c|c|c|c|c|}
\hline Parameter & No. & Median & IQR & $P_{\dagger}^{\dagger}$ \\
\hline \multicolumn{5}{|c|}{ Maternal HIV RNA viral load (copies/mL) } \\
\hline Enrollment $\$$ & 93 & 16,409 & $3,548-52,967$ & - \\
\hline Delivery & 194 & 17,515 & $4,800-69,492$ & 0.540 \\
\hline $\begin{array}{l}\text { One-month } \\
\text { post-delivery }\end{array}$ & 156 & 24,589 & $6,017-81,250$ & 0.215 \\
\hline \multicolumn{5}{|c|}{$\mathrm{CD} 4$ cell counts $($ cells $/ \mu \mathrm{L})$} \\
\hline Enrollment & 95 & 492 & $329-634$ & - \\
\hline $\begin{array}{l}\text { One-month } \\
\text { post-delivery }\end{array}$ & 84 & 635 & $460-827$ & $<0.001$ \\
\hline CD8 counts (cells/ & & & & \\
\hline Enrollment & 95 & 726 & $547-935$ & - \\
\hline $\begin{array}{l}\text { One-month } \\
\text { post-delivery }\end{array}$ & 84 & 1,063 & $787-1,257$ & $<0.001$ \\
\hline \multicolumn{5}{|c|}{ Ratio of CD4:CD8 cell counts } \\
\hline Enrollment & 95 & 0.64 & $0.49-0.86$ & - \\
\hline $\begin{array}{l}\text { One-month } \\
\text { post-delivery }\end{array}$ & 84 & 0.595 & $0.45-0.775$ & 0.001 \\
\hline
\end{tabular}

delivery this percentage decreased to $7 \%(P<0.001$, by McNemar's chi-square test for paired observations). The increase in CD4 cell counts was accompanied by a slightly larger increase in CD8 cell counts, leading to a small but significant decrease in the CD4:CD8 ratio $(P=0.003)$ (Table 3$)$.

Impact of sd-NVP administration on MTCT of HIV and development of resistance mutations. Although, $84 \%$ of the women reported taking their NVP tablet at the onset of labor and all infants received a NVP dose during the first 72 hours of life, no evidence was found of differences between maternal HIV RNA viral load at enrollment and at delivery (Table 3). Sequencing a region in the HIV RT gene showed that $22 \%$ (9 of 41) of sequences amplified harbored at least one highlevel resistance mutation, with two sequences harboring two resistance mutations. $\mathrm{K} 103 \mathrm{~N}$ was the most prevalent mutation, as described by others. ${ }^{24}$ All viruses studied were subtype C. ${ }^{25}$

Mother-to-child transmission of HIV. Results assessing MTCT of HIV infants at one month of age were available for $77 \%$ of live births where HIV DNA was detected in $12.4 \%$ of infants as described previously. ${ }^{21}$ From those infants who were negative for HIV DNA at one month of age and for whom HIV testing was available at 12 months of age, an additional $15.1 \%$ (14 of 93 ) (95\% CI $=7.6-22.4)$ were HIV DNA positive. Overall risk of mother-to-child transmission of HIV during the first year of life was calculated to be $25.6 \%$.

Infant mortality was evaluated in babies born to HIVinfected mothers according to the HIV status of the infant at one month of age. During the first year of life, 26.3\% (5 of 19) of HIV-positive infants died compared with $3.7 \%$ (5 of 134) HIV-negative infants $(P=0.003)$ (Table 4$)$. For those 46 with no HIV test available at one month of age, there were five deaths $(10.9 \%)$ over the first year of life.

\section{DISCUSSION}

This study assessed the impact of maternal HIV infection on birth outcomes and infant mortality in a rural area of southern Mozambique. It also described viral and immunologic parameters of HIV infection in pregnant women and their evolution in the postpartum. The findings of this study show that anemia at delivery was more common among HIV-positive women than in HIV-negative women. No statistically significant differences in adverse pregnancy outcomes, such as low birth weight, 
TABLE 4

HIV status and mortality in infants born to HIV-positive women*

\begin{tabular}{lcccc}
\hline \multicolumn{1}{c}{ Status } & $\mathrm{n} / \mathrm{N}$ & $\%$ & $95 \% \mathrm{CI}$ & $P$ \\
\hline \multicolumn{3}{l}{ Sex of HIV-infected infants perinatally $\dagger$} \\
$\quad$ Male & $9 / 79$ & 11.4 & $4.2-18.6$ & 0.690 \\
Female & $10 / 74$ & 13.5 & $5.5-21.5$ & \\
Infant mortality & & & & \\
HIV+ & $5 / 19$ & 26.3 & $4.5-48$ & $0.003 \ddagger$ \\
HIV- & $5 / 134$ & 3.7 & $0.4-7$ & \\
\hline
\end{tabular}

* HIV = human immunodeficiency virus; $\mathrm{CI}=$ confidence interval.

$\dagger$ Perinatal period covers the time until 4-6 weeks of age.

$\$$ By Fisher's exact test.

spontaneous abortions, preterm delivery, and stillbirth, were observed between HIV-positive and HIV-negative women. However, infants born to HIV-positive women had more than twice a higher risk of death than infants born to HIV-negative women. Several studies from sub-Saharan Africa suggest that HIV-infected mothers may be at increased risk of adverse pregnancy outcomes, especially for those women with anemia $^{26}$ or CD 4 cell counts less than 200 cells/ $\mu$ L. ${ }^{9,27}$

In this study the observed rates of adverse pregnancy outcomes in the HIV-positive and HIV-negative women were similar to those found in other studies. ${ }^{9,12}$ No statistically significant differences were found in the comparison between HIV-positive and HIV-negative women for low birth weight, spontaneous abortion, premature delivery, or stillbirth rates. However, there was a trend towards increased rates among HIV-positive women for most of these outcomes. The lack of statistical significance of these trends is likely caused by the small sample size.

The prevalence of anemia at delivery was significantly higher among HIV-infected women (51.3\%) than among HIV-negative women (35.4\%), which indicated that HIV infection was likely to be an etiologic factor of maternal anemia. Both HIV and malaria infection have been shown to be independent risk factors for maternal anemia. ${ }^{26,28,29}$ This finding is consistent with reported prevalence of anemia in pregnant African women ranging from $50 \%$ to $80 \%$, irrespective of HIV status. ${ }^{30,31}$

Maternal HIV infection was significantly associated with an excess infant mortality regardless of the HIV infection status of the infant. These findings are consistent with those of previous studies that have described maternal HIV infection as a risk factor for infant mortality. ${ }^{5,15,18,32}$ Several known risk factors for infant mortality associated with HIV infection include low birth weight, maternal anemia, maternal CD4 cell counts less than 200 cells/ $\mu \mathrm{L}$, or high maternal HIV RNA viral load in HIV-infected women. ${ }^{5,33,34} \mathrm{~A}$ recent community-based study in Rakai, Uganda ${ }^{18}$ showed two-year mortality rates of 540, 166, and 128 per thousand child-years among HIV-infected children, HIV-negative children of HIV-positive mothers, and HIV-negative children of HIV-negative women, respectively. Interestingly, maternal HIV infection was strongly associated with post-neonatal death but not with early and late neonatal death. Early and late neonatal death have been shown to be related to low birth weight and congenital malformations regardless of HIV status of the mother. ${ }^{26,35}$ Undeniably, infant mortality is complex and multifactorial, and risk factors change with age of the infant. This complexity must be taken into account when optimizing targets for implementation of public health interventions to reduce infant mortality.
The study began recruitment when VCT was implemented at Manhiça District Hospital. During the second year of the study, and probably because of a change of perception towards HIV testing, the percentage of women refusing counseling and testing decreased considerably, and the proportion of women positive for HIV increased significantly from $18.2 \%$ to $29 \%$. Prevalence of HIV is increasing in the region, ${ }^{1,25}$ and improved compliance for HIV testing may also lead to test acceptance by patients with higher risk of being HIV positive. Although HIV incidence data is not available in this population, almost $20 \%$ of the women in whom recent HIV infections where analyzed had contracted the infection within the previous six months. This finding suggests rapid spread of the HIV epidemic in this area over recent years. ${ }^{25}$ Consistent with previous reports, HIV-infected women were significantly older than HIV-negative women, which may be explained by the HIV epidemic history in southern Africa. Furthermore, malnutrition, syphilis, and malaria, although not significantly associated in this study with HIV infection, appeared to be more common among HIV-infected women. This finding is consistent with compiled evidence reporting the synergistic effect of HIV infection with other infectious diseases and deficiency status. ${ }^{36,37}$

As reported in a previous study, the rate of MTCT of HIV by 4-6 weeks of age found in this population was $12.4 \% .{ }^{21}$ This finding is similar to the $14.8 \%$ rate observed in KwaZulu Natal, South Africa by eight weeks of age ${ }^{9}$ where the routine administration of NVP was also unsupervised. ${ }^{6}$ Furthermore, in this study the rate of MTCT in the first year of life (excluding perinatal transmission) was $15.1 \%$, which highlights the urgent need to address the issue of breastfeeding transmission, including evaluation of the use of antiretroviral prophylaxis in the infant or the mother during breastfeeding., 48,39

The NVP resistance rate of $22 \%$ is low compared with published studies showing $69 \%$ mutation rates, ${ }^{24,40}$ particularly given the fact that it has been suggested that subtype $\mathrm{C}$ may be associated with detection of increased NVP-resistance mutations after sd-NVP when compared with A and D subtypes. ${ }^{41}$ The fact that we did not observe a significant decrease in maternal HIV RNA viral load between recruitment and delivery but rather a tendency towards an increase, as well as the surprisingly low resistance rate to NVP, suggests inadequate compliance with sd-NVP in this cohort despite high selfreported adherence. Some increase in HIV RNA viral load might have been explained by HIV disease progression. Recent reports in various sub-Saharan African countries have suggested that there may be an important gap between the efficacy of sd-NVP in the prevention of MTCT of HIV in a clinical trial setting and its effectiveness when implemented under real life conditions. ${ }^{42-44}$ Difficulties are likely to be caused by improper self-administration of NVP and poor compliance.

This study provides further evidence to support public health efforts in improving deployment of antiretroviral therapy during pregnancy and infancy in breastfeeding populations. Maternal HIV infection jeopardizes infant survival during infancy. Thus, effective public health measures to reduce MTCT of HIV during the first year of life is urgently needed to avoid excess mortality in the child. In conclusion, the epidemiology of HIV infection is not homogeneous throughout sub-Saharan Africa, and assessment of the effect of HIV infection on pregnancy outcomes and infant survival 
in rural African populations is necessary to guide targeted public health actions.

Received September 15, 2008. Accepted for publication January 12, 2009.

Acknowledgments: We thank the women and children for their participation in the study and the VCT, field, clinic, and data management staff at the Centro de Investigaçao em Saúde de Manhiça, Mozambique for their continued dedication.

Financial support: This study was supported by grant SAF-05845 from the Spanish Ministry of Education and Science and grant 02-0 from the Banco de Bilbao, Vizcaya Argentaria Foundation. The Centro de Investigaçao em Saúde de Manhiça receives major core funding from the Spanish Agency for International Cooperation, and the VCT center at Manhiça District Hospital is supported by the Generalitat de Catalunya. Denise Naniche was supported by a grant from the Spanish Ministry of Education and Science (Ramón y Cajal). María Lahuerta was supported by a grant from the Hospital Clinic Institut d'Investigacions Biomèdiques August Pi i Sunyer.

Disclosure: The authors have no conflicts of interest

Authors' addresses: Denise Naniche, María Lahuerta, and Sergi Sanz, Barcelona Center for International Health Research, Hospital Clinic, Institut d'Investigacions Biomèdiques August $\mathrm{Pi}$ i Sunyer, Universitat de Barcelona, C/Villarroel, 170, Barcelona 08036, Spain, E-mails: dsuzanne@clinic.ub.es,mlahuerta@yahoo.com, and ssanz@clinic.ub.es. Anna Berenguera, Manhiça Health Research Centre, Street N12, Manhiça Village, C.P. 1929 Maputo, Mozambique. Azucena Bardají, John J. Aponte, Pedro L. Alonso, and Clara Menéndez, Barcelona Center for International Health Research, Hospital Clinic, Institut d'Investigacions Biomèdiques August Pi i Sunyer, Universitat de Barcelona, C/Villarroel, 170, Barcelona 08036, Spain, and Manhiça Health Research Centre, Street N12, Manhiça Village, C.P. 1929 Maputo, Mozambique, E-mails: abardaji@clinic.ub.es, john.aponte@ cresib.cat,palonso@clinic.ub.es, and menendez@clinic.ub.es. Inacio Mandomando, Manhiça Health Research Centre, Street N12, Manhiça Village, C.P. 1929 Maputo, Mozambique and Instituto Nacional da Saúde, Ministerio da Saúde, Av. Eduardo Mondlane 1008, Maputo, C.P. 264, Maputo, Mozambique, E-mail: inacio.mandomando@manhica .net. Betuel Sigauque, Manhiça Health Research Centre, Street N12, Manhiça Village, C.P. 1929 Maputo, Mozambique and Faculdade de Medicina da Universidade Eduardo Mondlane, Av. Salvador Allende, 702, Maputo, Mozambique, E-mail: betuel.sigauque@manhica.net.

Reprints requests: Denise Naniche, Barcelona Centre for International Health, Institut d'Investigacions Biomèdiques August Pi i Sunyer/ Hospital Clinic, Villarroel, 170 Barcelona, E-08036 Spain, E-mail: dsuzanne@clinic.ub.es.

\section{REFERENCES}

1. UNAIDS/WHO, 2008. Report on the Global AIDS Epidemic. Geneva: World Health Organization.

2. Coovadia H, 2004. Antiretroviral agents-how best to protect infants from HIV and save their mothers from AIDS. $N$ Engl $J$ Med 351: 289-292.

3. De Cock KM, Fowler MG, Mercier E, de Vincenzi I, Saba J, Hoff E, Alnwick DJ, Rogers M, Shaffer N, 2000. Prevention of mother-to-child HIV transmission in resource-poor countries: translating research into policy and practice. JAMA 283: $1175-1182$.

4. Kourtis AP, Lee FK, Abrams EJ, Jamieson DJ, Bulterys M, 2006. Mother-to-child transmission of HIV-1: timing and implications for prevention. Lancet Infect Dis 6: 726-732.

5. Newell ML, Coovadia H, Cortina-Borja M, Rollins N, Gaillard P, Dabis F, 2004. Mortality of infected and uninfected infants born to HIV-infected mothers in Africa: a pooled analysis. Lancet 364: 1236-1243.

6. Chi BH, Wang L, Read JS, Sheriff M, Fiscus S, Brown ER, Taha TE, Valentine M, Goldenberg R, 2005. Timing of maternal and neonatal dosing of nevirapine and the risk of mother-to-child transmission of HIV-1: HIVNET 024. AIDS 19: 1857-1864.
7. World Health Organization, 2006. Antiretroviral Drugs for Treating Pregnant Women and Preventing HIV Infection in Infants: Towards Universal Access. Recommendations for a Public Health Approach. Geneva: World Health Organization.

8. Braddick MR, Kreiss JK, Embree JB, Datta P, Ndinya-Achola JO, Pamba H, Maitha G, Roberts PL, Quinn TC, Holmes KK, 1990. Impact of maternal HIV infection on obstetrical and early neonatal outcome. AIDS 4: 1001-1005.

9. Rollins NC, Coovadia HM, Bland RM, Coutsoudis A, Bennish ML, Patel D, Newell ML, 2007. Pregnancy outcomes in HIVinfected and uninfected women in rural and urban South Africa. J Acquir Immune Defic Syndr 44: 321-328.

10. Temmerman M, Plummer FA, Mirza NB, Ndinya-Achola JO, Wamola IA, Nagelkerke N, Brunham RC, Piot P, 1990. Infection with HIV as a risk factor for adverse obstetrical outcome. AIDS 4: $1087-1093$.

11. van Eijk AM, Ayisi JG, Ter Kuile FO, Misore AO, Otieno JA, Kolczak MS, Kager PA, Steketee RW, Nahlen BL, 2002. Malaria and human immunodeficiency virus infection as risk factors for anemia in infants in Kisumu, western Kenya. Am J Trop Med Hyg 67: 44-53.

12. Brocklehurst P, French R, 1998. The association between maternal HIV infection and perinatal outcome: a systematic review of the literature and meta-analysis. Br J Obstet Gynaecol 105: 836-848.

13. Dreyfuss ML, Msamanga GI, Spiegelman D, Hunter DJ, Urassa EJ, Hertzmark E, Fawzi WW, 2001. Determinants of low birth weight among HIV-infected pregnant women in Tanzania. Am J Clin Nutr 74: 814-826.

14. Garrib A, Jaffar S, Knight S, Bradshaw D, Bennish ML, 2006. Rates and causes of child mortality in an area of high HIV prevalence in rural South Africa. Trop Med Int Health 11: 1841-1848.

15. Kuhn L, Kasonde P, Sinkala M, Kankasa C, Semrau K, Scott N, Tsai WY, Vermund SH, Aldrovandi GM, Thea DM, 2005. Does severity of HIV disease in HIV-infected mothers affect mortality and morbidity among their uninfected infants? Clin Infect Dis 41: 1654-1661.

16. Dabis F, Elenga N, Meda N, Leroy V, Viho I, Manigart O, DequaeMerchadou L, Msellati P, Sombie I, 2001. 18-Month mortality and perinatal exposure to zidovudine in west Africa. AIDS 15: 771-779.

17. Taha TE, Kumwenda NI, Broadhead RL, Hoover DR, Graham SM, Van Der Hoven L, Markakis D, Liomba GN, Chiphangwi JD, Miotti PG, 1999. Mortality after the first year of life among human immunodeficiency virus type 1-infected and uninfected children. Pediatr Infect Dis J 18: 689-694.

18. Brahmbhatt H, Kigozi G, Wabwire-Mangen F, Serwadda D, Lutalo T, Nalugoda F, Sewankambo N, Kiduggavu M, Wawer M, Gray R, 2006. Mortality in HIV-infected and uninfected children of HIV-infected and uninfected mothers in rural Uganda. J Acquir Immune Defic Syndr 41: 504-508.

19. Alonso PL, Saúte F, Aponte JJ, 2001. Manhiça DSS, Mozambique. Population. Health and Survival at INDEPTH Sites 1: 189-195.

20. Menendez C, Bardaji A, Sigauque B, Romagosa C, Sanz S, SerraCasas E, Macete E, Berenguera A, David C, Dobano C, Naniche D, Mayor A, Ordi J, Mandomando I, Aponte JJ, Mabunda S, Alonso PL, 2008. A randomized placebo-controlled trial of intermittent preventive treatment in pregnant women in the context of insecticide treated nets delivered through the antenatal clinic. PLoS One 3: e1934.

21. Naniche D, Lahuerta M, Bardaji A, Sigauque B, Romagosa C, Berenguera A, Mandomando I, David C, Sanz S, Aponte J, Ordi J, Alonso P, Menendez C, 2008. Mother-to-child transmission of HIV-1: association with malaria prevention, anaemia and placental malaria. HIV Med 9: 757-764.

22. Shafer RW, Eisen JA, Merigan TC, Katzenstein DA, 1997. Sequence and drug susceptibility of subtype C reverse transcriptase from human immunodeficiency virus type 1 seroconverters in Zimbabwe. J Virol 71: 5441-5448.

23. World Health Organization, 2006. Reproductive Health Indicators. Geneva: World Health Organization.

24. Eshleman SH, Guay LA, Mwatha A, Brown ER, Cunningham SP Musoke P, Mmiro F, Jackson JB, 2004. Characterization of nevirapine resistance mutations in women with subtype $\mathrm{A}$ vs. D HIV-1 6-8 weeks after single-dose nevirapine (HIVNET 012). J Acquir Immune Defic Syndr 35: 126-130. 
25. Lahuerta M, Aparicio E, Bardaji A, Marco S, Sacarlal J, Mandomando I, Alonso P, Martinez MA, Menendez C, Naniche D, 2008. Rapid spread and genetic diversification of HIV type 1 subtype $\mathrm{C}$ in a rural area of southern Mozambique. AIDS Res Hum Retroviruses 24: 327-335.

26. Steketee RW, Nahlen BL, Parise ME, Menendez C, 2001. The burden of malaria in pregnancy in malaria-endemic areas. $\mathrm{Am} \mathrm{J}$ Trop Med Hyg 64: 28-35.

27. Langston C, Lewis DE, Hammill HA, Popek EJ, Kozinetz CA, Kline MW, Hanson IC, Shearer WT, 1995. Excess intrauterine fetal demise associated with maternal human immunodeficiency virus infection. $J$ Infect Dis 172: 1451-1460.

28. Ayisi JG, van Eijk AM, ter Kuile FO, Kolczak MS, Otieno JA, Misore AO, Kager PA, Steketee RW, Nahlen BL, 2003. The effect of dual infection with HIV and malaria on pregnancy outcome in western Kenya. AIDS 17: 585-594.

29. Menendez C, Fleming AF, Alonso PL, 2000. Malaria-related anaemia. Parasitol Today 16: 469-476.

30. Belperio PS, Rhew DC, 2004. Prevalence and outcomes of anemia in individuals with human immunodeficiency virus: a systematic review of the literature. Am J Med 116 (Suppl 7A): 27S-43S.

31. Dairo MD, Lawoyin TO, Onadeko MO, Asekun-Olarinmoye EO, Adeniji AO, 2005. HIV as an additional risk factors for anaemia in pregnancy: evidence from primary care level in Ibadan, Southwestern Nigeria. Afr J Med Sci 34: 275-279.

32. Verhoeff FH, Le Cessie S, Kalanda BF, Kazembe PN, Broadhead RL, Brabin BJ, 2004. Post-neonatal infant mortality in Malawi: the importance of maternal health. Ann Trop Paediatr 24: 161-169.

33. McCormick MC, 1985. The contribution of low birth weight to infant mortality and childhood morbidity. $N$ Engl J Med 312: 82-90.

34. Obimbo EM, Mbori-Ngacha DA, Ochieng JO, Richardson BA, Otieno PA, Bosire R, Farquhar C, Overbaugh J, John-Stewart GC, 2004. Predictors of early mortality in a cohort of human immunodeficiency virus type 1-infected African children. Pediatr Infect Dis J 23: 536-543.

35. Taha TE, Dallabetta GA, Canner JK, Chiphangwi JD, Liomba G, Hoover DR, Miotti PG, 1995. The effect of human immunodefi- ciency virus infection on birthweight, and infant and child mortality in urban Malawi. Int J Epidemiol 24: 1022-1029.

36. Steketee RW, 2003. Pregnancy, nutrition and parasitic diseases. J Nutr 133: 1661S-1667S.

37. Steketee RW, Wirima JJ, Bloland PB, Chilima B, Mermin JH, Chitsulo L, Breman JG, 1996. Impairment of a pregnant woman's acquired ability to limit Plasmodium falciparum by infection with human immunodeficiency virus type-1. Am J Trop Med Hyg 55: 42-49.

38. Fawzi W, Msamanga G, Spiegelman D, Renjifo B, Bang H, Kapiga S, Coley J, Hertzmark E, Essex M, Hunter D, 2002. Transmission of HIV-1 through breastfeeding among women in Dar es Salaam, Tanzania. J Acquir Immune Defic Syndr 31: 331-338.

39. Miotti PG, Taha TE, Kumwenda NI, Broadhead R, Mtimavalye LA, Van der Hoeven L, Chiphangwi JD, Liomba G, Biggar RJ, 1999. HIV transmission through breastfeeding: a study in Malawi. JAMA 282: 744-749.

40. Eshleman SH, Hoover DR, Chen S, Hudelson SE, Guay LA, Mwatha A, Fiscus SA, Mmiro F, Musoke P, Jackson JB, Kumwenda N, Taha T, 2005. Resistance after single-dose nevirapine prophylaxis emerges in a high proportion of Malawian newborns. AIDS 19: 2167-2169.

41. Eshleman SH, Hoover DR, Chen S, Hudelson SE, Guay LA, Mwatha A, Fiscus SA, Mmiro F, Musoke P, Jackson JB, Kumwenda N,Taha T, 2005. Nevirapine (NVP) resistance in women with HIV-1 subtype C, compared with subtypes A and D, after the administration of single-dose NVP. J Infect Dis 192: 30-36.

42. Delva W, Draper B, Temmerman M, 2006. Implementation of single-dose nevirapine for prevention of MTCT of HIV-lessons from Cape Town. S Afr Med J 96: 706, 708-709.

43. Perez F, Mukotekwa T, Miller A, Orne-Gliemann J, Glenshaw M, Chitsike I, Dabis F, 2004. Implementing a rural programme of prevention of mother-to-child transmission of HIV in Zimbabwe: first 18 months of experience. Trop Med Int Health 9: 774-783.

44. Quaghebeur A, Mutunga L, Mwanyumba F, Mandaliya K, Verhofstede C, Temmerman M, 2004. Low efficacy of nevirapine (HIVNET012) in preventing perinatal HIV-1 transmission in a real-life situation. AIDS 18: 1854-1856. 